\title{
Erdbebenvorsorge in der Schweiz
}

\section{Journal Article}

\section{Author(s):}

Wenk, Thomas (D); Beyer, Katrin; Duvernay, Blaise

Publication date:

2015-04

\section{Permanent link:}

https://doi.org/10.3929/ethz-a-010396057

Rights / license:

In Copyright - Non-Commercial Use Permitted

Originally published in:

Bauingenieur 90 


\title{
Erdbebenvorsorge in der Schweiz
}

\author{
T. Wenk, K. Beyer, B. Duvernay
}

\section{Prof. Dr. Dr. h.c. Hugo Bachmann zum 80. Geburtstag gewidmet}

\section{Einleitung}

Wie kein anderer hat sich Prof. Dr. Dr. h.c.

Hugo Bachmann während der letzten 30 Jahre tatkräftig und unermüdlich für die Erdbebenvorsorge in der Schweiz eingesetzt (Bild 1). Sein baldiger 80 . Geburtstag am 27. September 2015 soll Anlass sein, die Fortschritte der Erdbebenvorsorge in den letzten Jahren kurz darzustellen. Rückblickend lässt sich feststellen, dass die SGEB-Publikation „Handlungsbedarf” [1] aus dem Jahre 1998 das Schlüsseldokument und der Wendepunkt in der Erdbebenvorsorge in der Schweiz geworden ist. Während vorher die Erdbebensi-

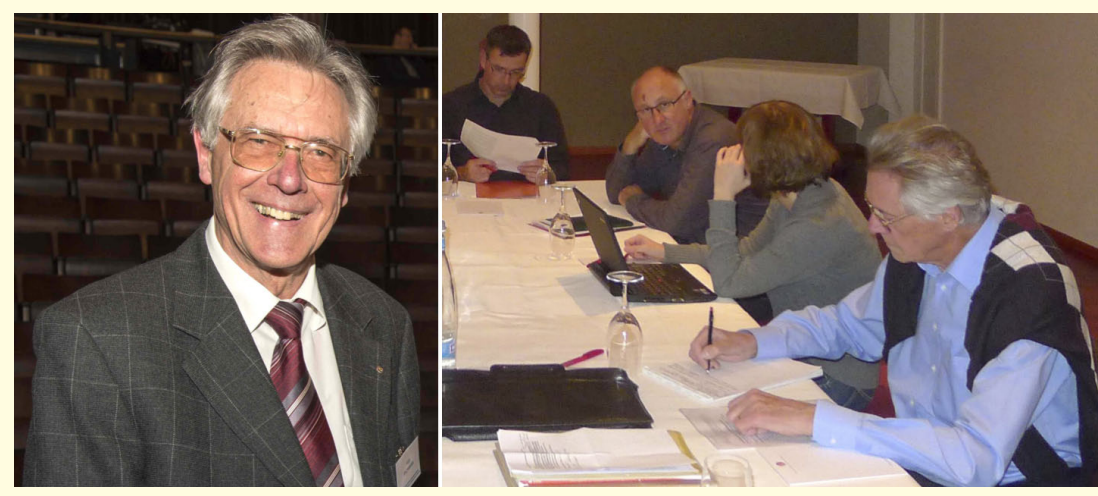

Bild 1. Prof. Dr. Dr. h.c. Hugo Bachmann an der Universität Fribourg im Januar 2015 (links, Foto: M. Deuring) und an der SGEB-Vorstandssitzung neben Katrin Beyer, Donat Fäh und Blaise Duvernay im November 2012 (rechts). cherung von Neubauten oft vernachlässigt wurde und die Überprüfung der Erdbebensicherheit bestehender Bauten kein Thema war, sind danach klare Fortschritte zu erkennen. Der „Handlungsbedarf” wurde auf Anregung und unter Federführung von Prof. Bachmann vom erweiterten Vorstand der Schweizer Gesellschaft für Erdbebeningenieurwesen und Baudynamik (SGEB) erarbeitet. Darin wurden die früheren Defizite der Erdbebenvorsorge systematisch und umfassend dargestellt.

\section{Koordinationsstelle des Bundes}

Ein erster wichtiger Erfolg des „Handlungsbedarfs“ war der Entscheid des Bundesrats vom 11. Dezember 2000, ein Maßnahmenprogramm zur Erdbebenvorsorge zu lancieren und die Koordinationsstelle des Bundes für Erdbebenvorsorge zu schaffen [2]. Die Koordinationsstelle hat seither zahlreiche Publikationen als unentbehrliche Hilfsmittel für die Praxis herausgegeben. Ferner unterstützt sie die Erstellung der Karten der Baugrundklassen gemäß Norm SIA 261, die zusammen mit spektralen Mikrozonierungskarten auf dem geologischen Datenviewer http://map.bafu.admin.ch zur Verfügung stehen [3].

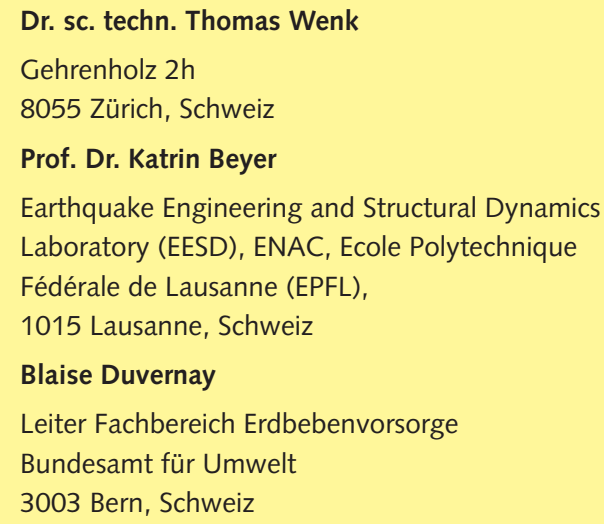

\section{Unterricht und Forschung}

An den beiden ETH‘s in Lausanne und Zürich führen größere, international renommierte Forschungsgruppen in Erdbebeningenieurwesen und Baudynamik die von Prof. Bachmann vor 40 Jahren in der Schweiz initiierte Forschungsrichtung erfolgreich weiter (Bild 2). Ein wichtiger Meilenstein war die Veröffentlichung des weltweit ersten Lehrbuchs zur Methode der Kapazitätsbemessung im Jahre 1990 [4]. Der traditionell praxisnahe Unterricht in diesen Disziplinen findet weiterhin großen Anklang bei den Studierenden in Bauingenieurwissenschaften und neuerdings auch bei denjenigen in Architektur. An den beiden Fachhochschulen in Fribourg und Luzern werden jährliche Weiterbildungskurse in Erdbebeningenieurwesen angeboten, die mit dem Certificate of Advanced Studies (CAS) abgeschlossen werden können.

\section{Tragwerksnormen}

\subsection{Normen für Neubauten}

Die Einführung der neuen Tragwerksnormen SIA 260 bis SIA 267 im Jahre 2003 markiert einen weiteren Wendepunkt in der Erdbebenvorsorge. Durch die sehr starke Erhöhung der Erdbebeneinwirkung gegenüber früheren Normengenerationen erhielt das Erdbeben einen bedeutenderen Stellenwert in der Praxis. Auch in der niedrigsten Zone Z1 wurde nun die Bemessungssituation Erdbeben oft maßgebend. Bei Neubauten bleiben jedoch die Mehrkosten der Erdbebensicherung in der Regel vernachlässigbar, falls die Grundsätze des erdbebengerechten Entwurfs bereits von der frühen Entwurfsphase an berücksichtigt werden [5]. In der kürzlich abgeschlossen Teilrevision dieser Normen erfolgten kleinere Anpassungen der Erdbebenkapitel an die heutige Fassung des Eurocodes 8.

\subsection{Normen für bestehende Bauten}

Die Ertüchtigung von bestehenden Bauten auf das im Jahre 2003 stark erhöhte Anforderungsniveau für Neubauten kann zu erheblichen Kosten führen. Das Merkblatt SIA 2018 [6] erlaubt als erste Erdbebennorm, bei bestehenden Ge- 

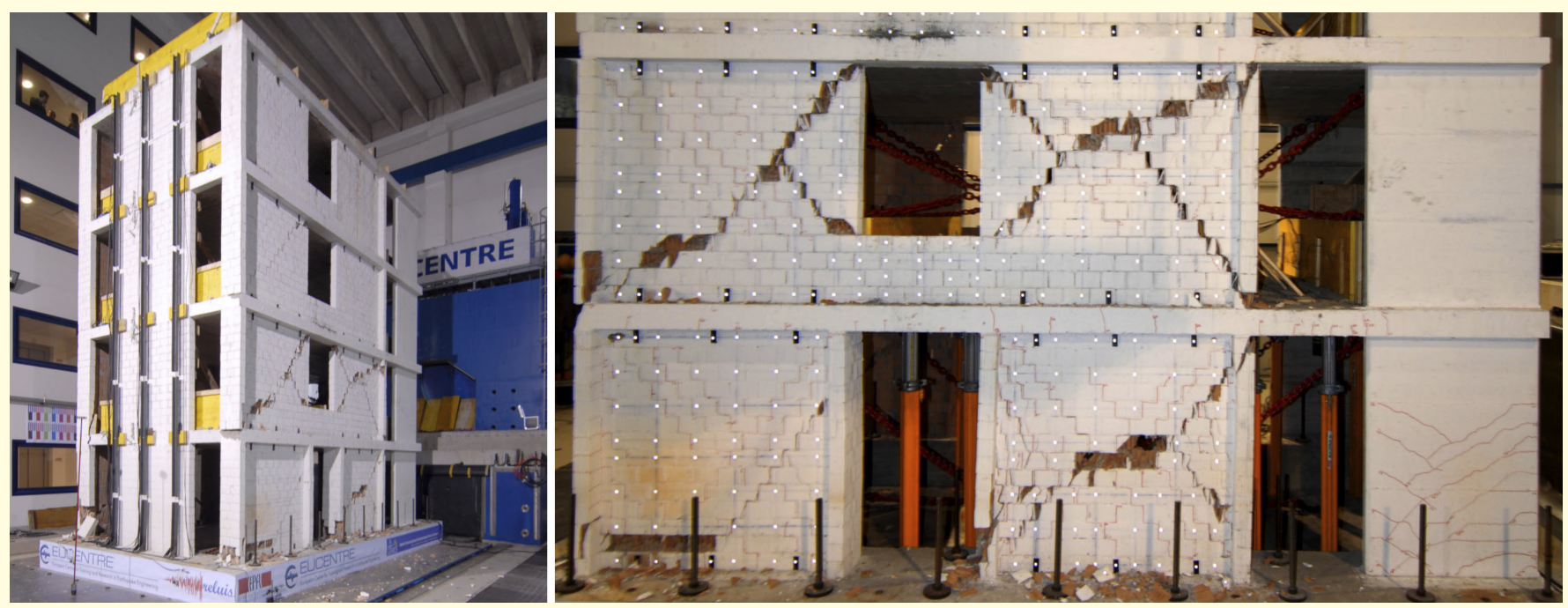

Bild 2. Rütteltischversuch an einem viergeschossigen Gebäude im Maßstab 1:2 mit Mauerwerks- und Stahlbetontragwänden (links), Detail der unteren zwei Geschosse, die in Längsrichtung angeregt wurden (rechts) [9].
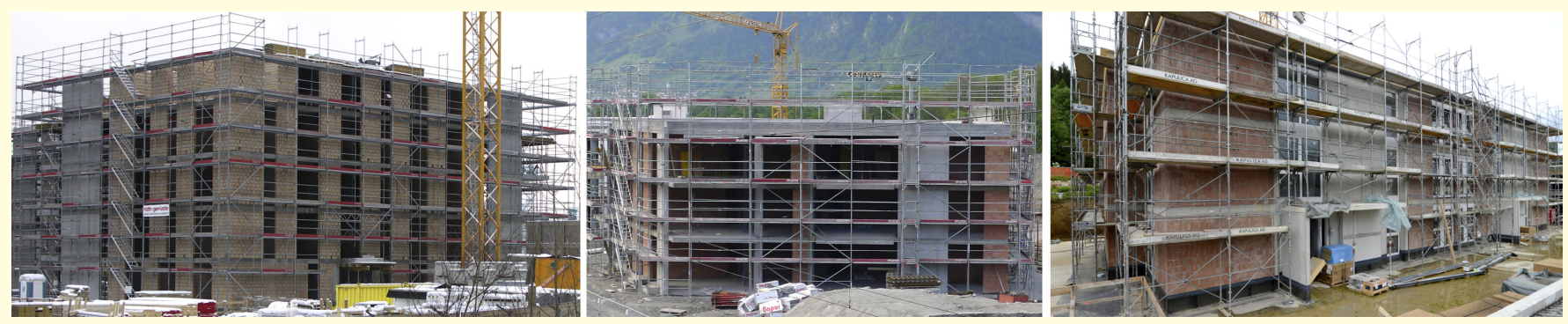

Bild 3. Baustellen von Wohnungsbauten mit Mauerwerks- und Stahlbetontragwänden in Erdbebenzone Z1 (links), in Erdbebenzone Z2 (Mitte) und in Erdbebenzone $\mathrm{Z3a}$ (rechts).

bäuden ein niedrigeres Sicherheitsniveau zu akzeptieren, solange gewisse Minimalanforderung bezüglich der individuellen und kollektiven Personenrisiken erfüllt sind. Zur Zeit wird das Merkblatt SIA 2018 überarbeitet. Es soll demnächst in die Erhaltungsnorm SIA 269/8 im Rahmen der Normenreihe SIA 269 „Erhaltung von Tragwerken“ überführt werden [7].

\subsection{Verbindlichkeit der Normen}

Bei der rechtlichen Verbindlichkeit der Erdbebenbestimmungen in den SIA-Tragwerksnormen sind schrittweise Fortschritte erzielt worden. Neben dem Bund führen nun mehrere Kantone die entsprechenden Normen inkl. Merk-

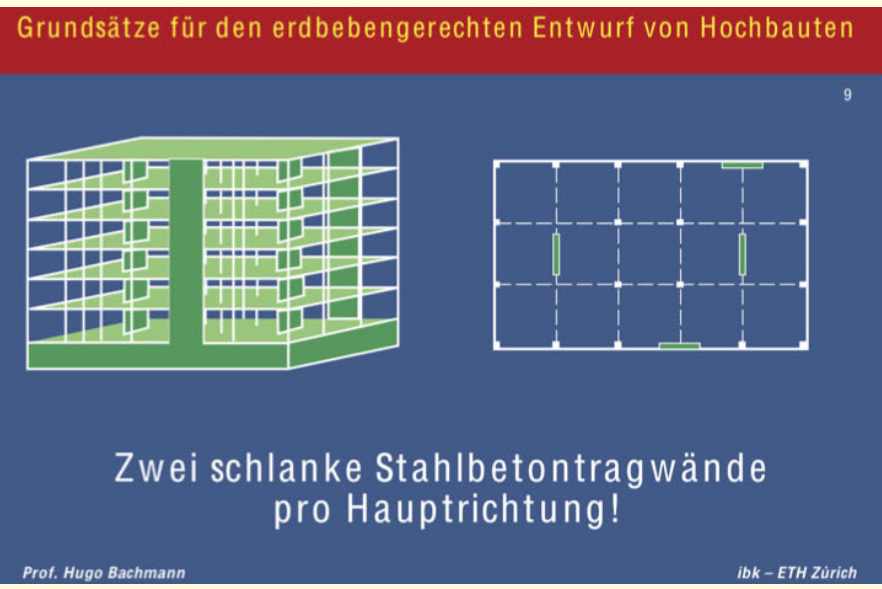

blatt SIA 2018 namentlich im Baurecht als verbindlich auf, seit kurzem auch der Kanton Zürich [8]. Abgesehen davon sind diese Bestimmungen bereits aufgrund der Generalklauseln zur Einhaltung der anerkannten Regeln der Baukunde verbindlich. Ein Nachholbedarf besteht weiterhin bei einigen Kantonen bezüglich verstärkter Kontrollen im Baubewilligungsverfahren, da sich das Prinzip der Eigenverantwortung als ungenügend erwiesen hat.

\section{Gebäude}

\subsection{Neubauten}

Ein Blick auf heutige Baustellen im Wohnungsbau zeigt, dass wesentliche Fortschritte erzielt worden sind (Bild 3).

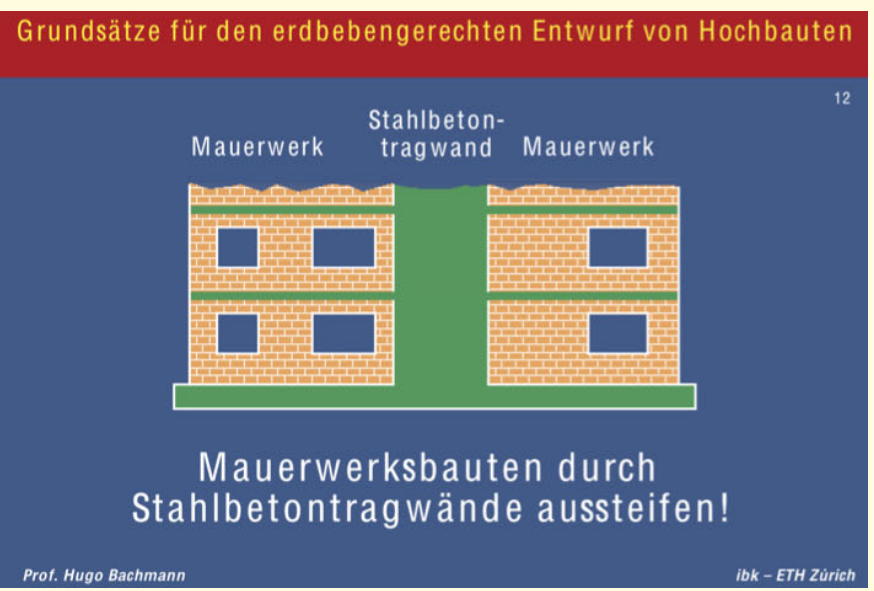

Bild 4. Zwei wichtige Grundsätze des erdbebengerechten Entwurfs von Gebäuden aus [5]. 

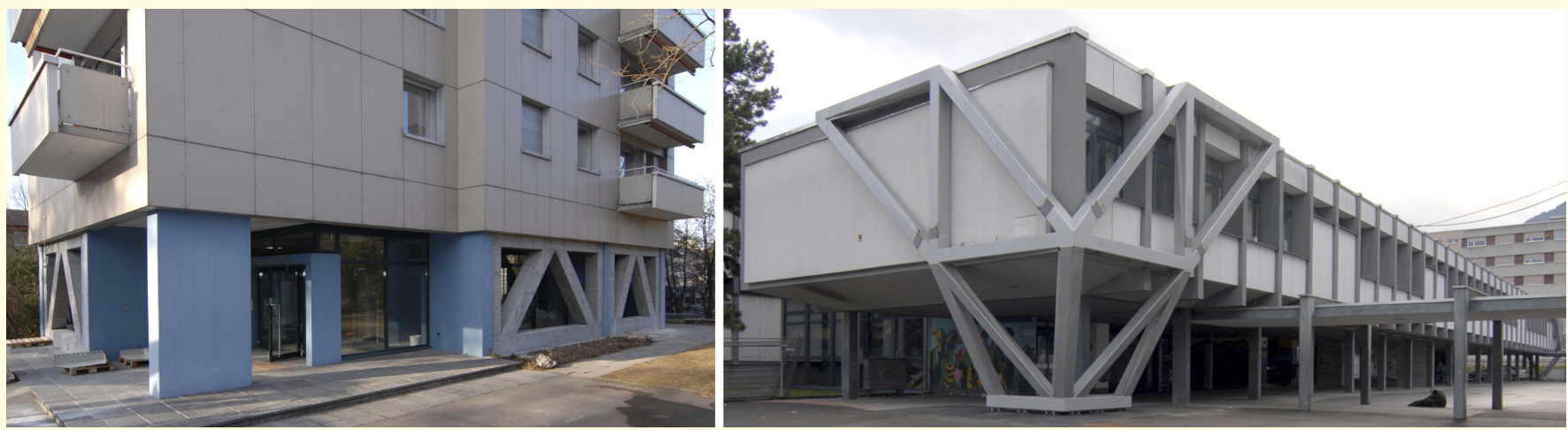

Bild 5. Erdbebenertüchtigung eines Wohngebäudes in Birsfelden bei Basel durch Stahlbetonfachwerke im Erdgeschoss (links) und eines Schulgebäudes in Monthey im Wallis durch räumliche Stahlfachwerke (rechts).

Oft ist bei Mauerwerksgebäuden zu sehen, dass Prof. Bachmann's Grundsätze des erdbebengerechten Entwurfs beachtet werden [5], insbesondere seine beiden Grundsätze: „Zwei schlanke Stahlbetontragwände pro Hauptrichtung!“ (Bild 4, links) und „Mauerwerksbauten durch Stahlbetontragwände aussteifen!“ (Bild 4, rechts). Bemessungsregeln für solche Verbundkonstruktionen aus Mauerwerk und Stahlbeton sind in Vorbereitung [9].

Das Aussteifungssystem heutiger Geschäftshäuser folgt in der Regel dem Grundsatz „Zwei schlanke Stahlbetontragwände pro Hauptrichtung!“, wobei die Stahlbetontragwände durchgehend über alle Geschosse laufen. Die früher anzutreffenden horizontal weichen Erdgeschosse sind verschwunden.

\subsection{Bestehende Gebäude}

Bei den bestehenden Gebäuden verläuft der Fortschritt langsamer, da die Überprüfung der Erdbebensicherheit anspruchsvoll und aufwendig ist. Im „Handlungsbedarf” [1] wurde bereits erkannt, dass dieser Aspekt für das Erdbebenrisiko in der Schweiz maßgebend ist: „95\% der bestehenden Gebäude in der Schweiz wurden nicht oder nur nach veralteten Regeln für Erdbeben bemessen. Sie haben eine nicht näher bekannte, oft aber ungenügende Erdbe- bensicherheit.“ Effektiv hat sich gezeigt, dass unter Berücksichtigung der risikobasierten Kriterien des Merkblatts SIA 2018 sowie moderner Tragwerksmodellierungen und Berechnungsmethoden nur ein kleiner Teil der Gebäude ertüchtigt werden muss, wobei genaue Zahlen fehlen. Bild 5 zeigt zwei Beispiele ausgeführter Erdbebenertüchtigung von Gebäuden. Ein Wohngebäude in Birsfelden bei Basel wurde durch Stahlbetonfachwerke im Erdgeschoss verstärkt (Bild 5, links) und ein Schulgebäude in Monthey im Wallis durch räumliche Stahlfachwerke (Bild 5, rechts). Das Schulgebäude wurde mit dem Architektur- und Ingenieurpreis erdbebensicheres Bauen 2012 der Stiftung für Baudynamik und Erdbebeningenieurwesen ausgezeichnet [10].

\subsection{Denkmalgeschützte Bauten}

Bei denkmalgeschützten Bauten, insbesondere beim historischen Mauerwerk, besteht eine gewisse Zurückhaltung, die Erdbebenproblematik anzugehen. Es wird meist befürchtet, dass die Erdbebeningenieure die Absicht hegen, durch irreversible bauliche Eingriffe das Denkmal zu zerstören. Einzelne denkmalgeschützte Bauten sind erfolgreich ertüchtigt worden, so zum Beispiel das Landesmuseum in Zürich (Bild 6). Eine interdisziplinäre Arbeitsgruppe mit Vertretern der Denkmalpflege, der Architektur und
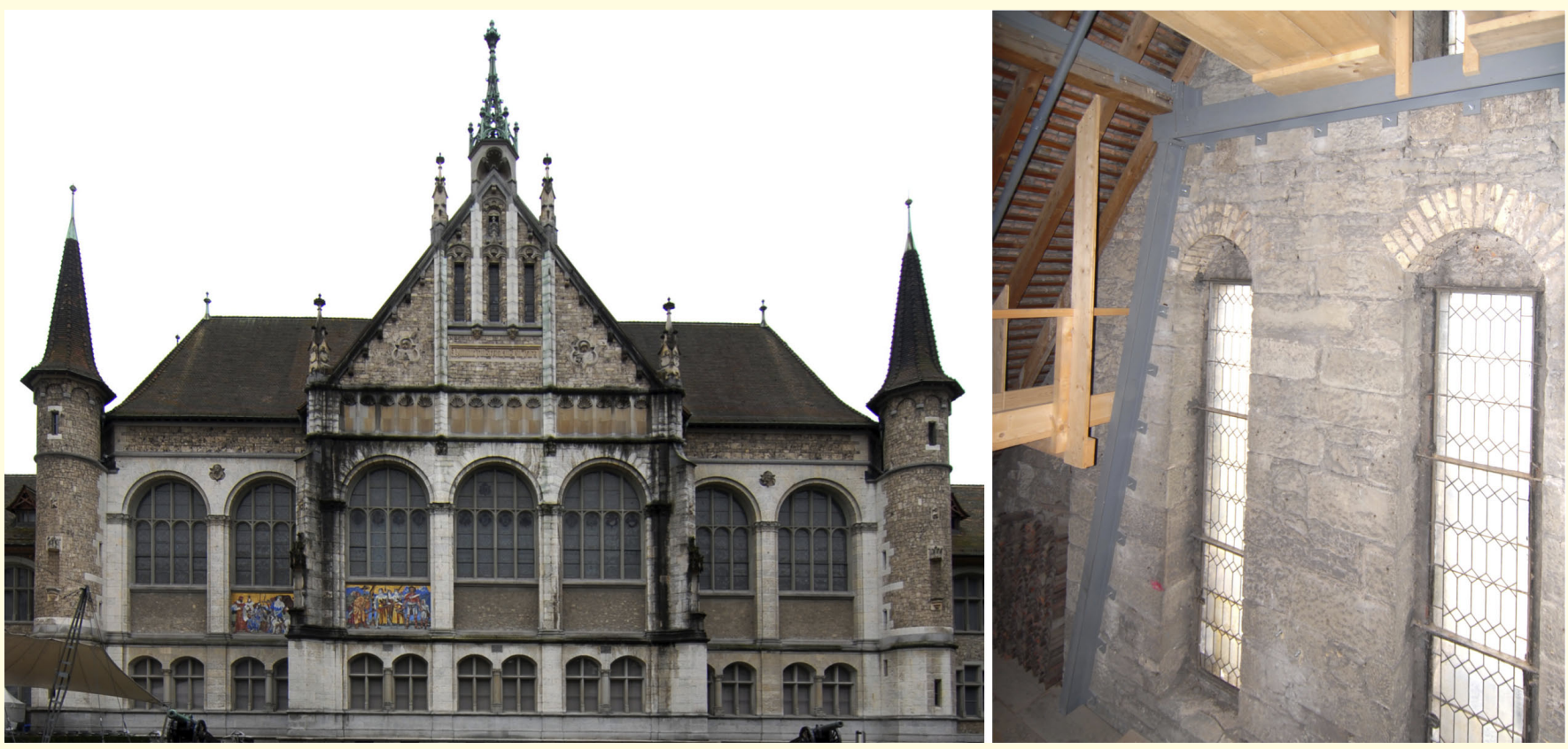

Bild 6. Erdbebenertüchtigung des Landesmuseums in Zürich: Ansicht an die Längsgiebelwand der Ruhmeshalle (links), Stahlrahmen auf der Innenseite der Längsgiebelwand zur Sicherung quer zur Wandebene (rechts). 

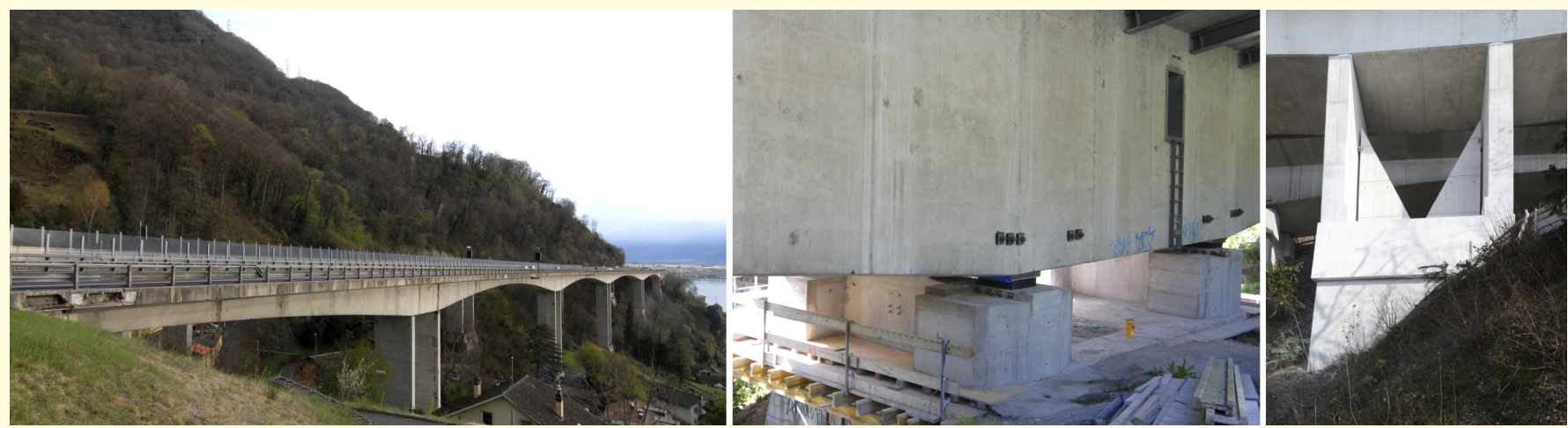

Bild 7. Erdbebenertüchtigung der Zwillingsviadukte von Chillon (links), seismische Isolation mit Blei-Gummilagern auf einer niedrigen Doppelstütze (Mitte), Stahlbeton-Ummantelung einer höheren Doppelstütze (rechts).

des Erdbebeningenieurwesens erarbeitet zur Zeit unter Leitung der Koordinationsstelle des Bundes für Erdbebenvorsorge eine Wegleitung für die Praxis.

\section{Brücken}

In den Jahren 2005 und 2006 wurde die 1. Stufe eines zweistufigen Verfahrens zur Überprüfung der Erdbebensicherheit bei allen rund 4.000 Brücken des Nationalstraßennetzes durchgeführt [11]. Rund ein Fünftel davon musste anschließend die umfassendere 2. Stufe durchlaufen. Bei etwa einem Fünftel der Überprüfungen in der 2. Stufe haben sich Erdbebenertüchtigungsmaßnahmen als erforderlich gezeigt, die meistens im Rahmen der planmäßigen Unterhaltsabschnitte ausgeführt werden, um Synergien mit allgemeinen Erhaltungsmaßnahmen zu nutzen. Auch einige Kantone, darunter Luzern und Zürich, haben ihre Kantonsstraßenbrücken nach dem zweistufigen Verfahren des Bundesamts für Straßen überprüft. Bild 7 zeigt als Beispiel für ausgeführte Erdbebenertüchtigungen den Viadukt von Chillon entlang des Genfersees, bei dem im Ist-Zustand die Erdbebensicherheit mehrerer Brückenabschnitte in Längsrichtung ungenügend war. Als Ertüchtigungsmaßnahme wurde das Verformungsvermögen in Brückenlängsrichtung bei den niedrigen Stützen durch seismische Isolation und bei den etwas höheren Stützen durch Stahlbeton-Ummantelung stark erhöht, während die hohen Stützen bereits im
Ist-Zustand ein genügendes Verformungsvermögen aufwiesen.

Bestehende Eisenbahnbrücken sind in der Regel weniger kritisch bezüglich Erdbeben als Strassenbrücken. Da sie für höhere Horizontalkräfte (Anfahr-, Brems-, Schlinger- und Zentrifugalkräfte) bemessen wurden, weisen sie gegenüber Strassenbrücken ein besseres Erdbebenverhalten auf. Zur Zeit wird das zweistufige Verfahren für Strassenbrücken für Eisenbahnbrücken weiterentwickelt und bei Probeanwendungen getestet.

\section{Stauanlagen}

Im 2003 verlangte das Bundesamt für Energie für alle 206 Stauanlagen, die der direkten Aufsicht des Bundes unterstehen, eine Überprüfung der Erdbebensicherheit bis 2013. Die meisten Stauanlagen können im Ist-Zustand als genügend erdbebensicher akzeptiert werden. Bisher erfolgte eine umfangreiche Ertüchtigung der $86 \mathrm{~m}$ hohen und $460 \mathrm{~m}$ langen Bogenstaumauer Les Toules im Wallis mit bis zu $12 \mathrm{~m}$ dicken massiven Beton-Stützkörpern auf beiden Talseiten (Bild 8). Die $25 \mathrm{~m}$ hohe und $350 \mathrm{~m}$ lange, kombinierte Bogen- und Schwergewichtsstaumauer Illsee, ebenfalls im Wallis gelegen, wird zur Zeit ertüchtigt.

Ältere Flusskraftwerke weisen oft Mängel in der Erdbebensicherheit der Wehre auf vor allem quer zur Flussrichtung. Auf diese Problematik wurde bereits in [1] hingewiesen und
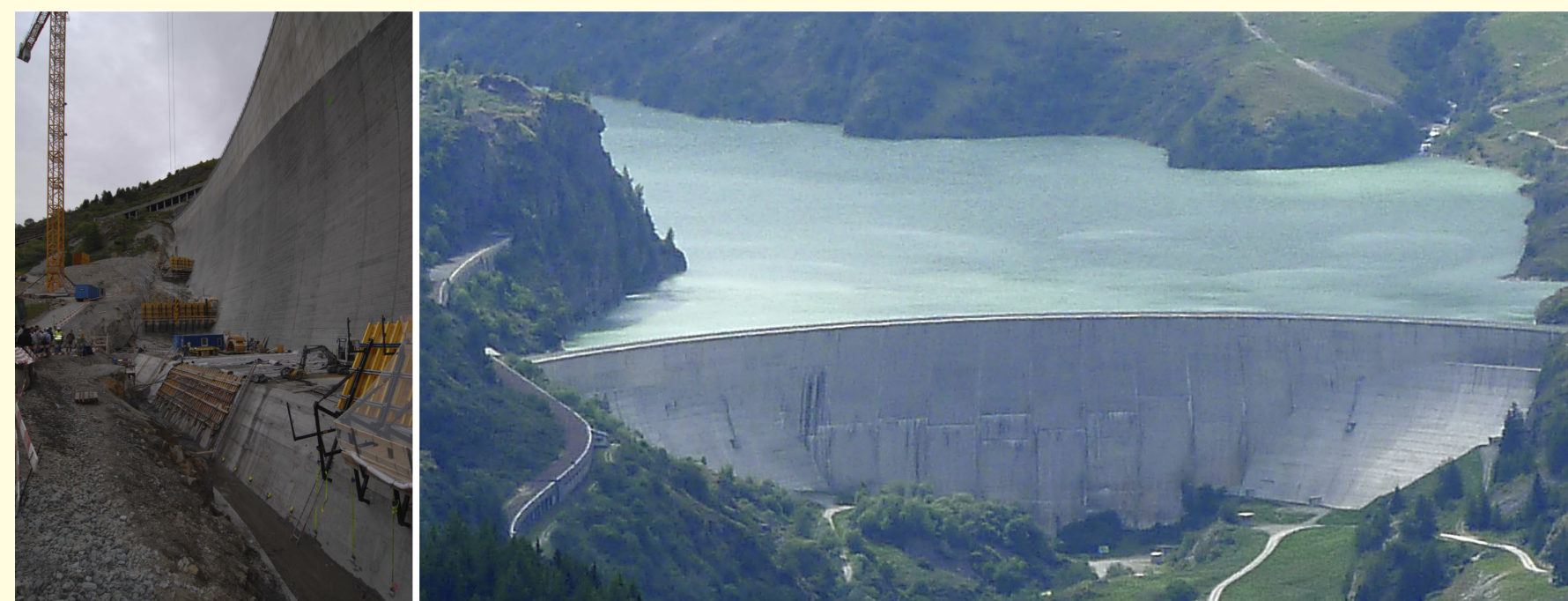

Bild 8. Erdbebenertüchtigung der Bogenstaumauer Les Toules im Wallis durch massiven Beton-Stützkörper auf der linken Talseiten (links) und mit den fertiggestellten Stützkörpern auf beiden Talseiten (rechts). 
als Folge wurden in den letzten Jahren einige Flusskraftwerke ertüchtigt.

\section{Erdbebenversicherung}

In den letzten 15 Jahren sind mehrere parlamentarische Vorstösse für eine obligatorische Erdbebenversicherung eingereicht worden, ohne dass es bisher zu einer Lösung gekommen wäre. Im letzten Jahr hat die Basellandschaftliche Kantonalbank mit einem innovativen Versicherungsmodell einen Schritt in Richtung eines Beinahe-Versicherungsobligatoriums gewagt. Gebäude bis zu einem Wert von 5 Mio. CHF, für welche die Basellandschaftliche Kantonalbank ein Hypothekardarlehen gewährt, werden ohne Zusatzprämie automatisch gegen Erdbebenschäden versichert [12]. Es ist zu erwarten, dass bald weitere Banken, insbesondere Kantonalbanken, ähnliche Versicherungsmodelle anbieten werden.

\section{SGEB}

Die Anzahl Mitglieder der Schweizer Gesellschaft für Erdbebeningenieurwesen und Baudynamik (SGEB) hat in den letzten Jahren erfreulich zugenommen. Ausgehend von 270 SGEB-Mitgliedern im Jahre 2001 sind heute 470 Mitglieder erreicht worden (Bild 9). Zum Vergleich sei die gegenläufige Entwicklung der Anzahl Mitglieder der Berufsgruppe Ingenieurbau (BGI) des Schweizerischen Ingenieur- und Architektenvereins (SIA) genannt, die in der gleichen Zeitspanne von 3800 auf 3400 zurückgegangen ist.

Der Haupttätigkeitsbereich der SGEB war in den letzten Jahren die Durchführung von Weiterbildungskursen für die Praxis. Mehrere Weiterbildungskurse pro Jahr wurden meist zusammen mit Partner-Organisationen angeboten.
Hinzu kommen die jährlichen Fachtagungen anlässlich der SGEB-Generalversammlung, die immer mehr Teilnehmer anziehen. Aufgrund der Antworten der Online-Mitgliederbefragung im 2013 werden in Zukunft vermehrt Weiterbildungskurse zur Problematik der bestehenden Bauten organisiert [13]. Der Jubilar leitet die sehr aktive SGEB-Arbeitsgruppe Öffentlichkeitsarbeit, die regelmäßig mit Beiträgen in den Medien auf Defizite in der Erdbebenvorsorge aufmerksam macht.

\section{Stiftung für Baudynamik und Erdbebeningenieurwesen}

Die im Jahre 2004 von Prof. Bachmann gegründete Stiftung für Baudynamik und Erdbebeningenieurwesen sensibilisiert sehr erfolgreich die Öffentlichkeit für die Belange der Erdbebenvorsorge, indem sie regelmäßig den Architekturund Ingenieurpreis erdbebensicheres Bauen [10] und den Innovationspreis Baudynamik verleiht. Stiftung und SGEB pflegen eine intensive und fruchtbare Zusammenarbeit, zum Beispiel bei der gemeinsamen Publikation von Faltblättern und Broschüren.

\section{Literatur}

[1] Bachmann, H.; Darbre, G. R.; Deichmann, N.; Koller, M. G.; Studer, J. A.; Tiniç, S.; Tissières, P.; Wenk, T.; Wieland, M.; Zwicky, P.: Handlungsbedarf von Behörden, Hochschulen, Industrie und Privaten zur Erdbebensicherung von Bauwerken in der Schweiz. SIA-Dokumentation D0150. Schweizerischer Ingenieur- und Architekten-Verein, Zürich 1998. www.sgeb.ch/fachpublikationen.html

[2] Bundesamt für Umwelt: Erdbebenvorsorge - Massnahmen des Bundes, Bericht an den Bundesrat, Standbericht und Planung für den Zeitraum 2013 bis 2016, Bern 2013.

[3] Duvernay, B.: Stand und Entwicklung der Erdbebenvorsorge in der Schweiz, D-A-CH-Tagung Erdbeben und Baudynamik, Hannover 2011.

[4] Paulay, T.; Bachmann, H.; Moser K.: Erdbebenbemessung von Stahlbetonhochbauten, Birkhäuser 1990

[5] Bachmann, H.: Erdbebengerechter Entwurf von Hochbauten - Grundsätze für Ingenieure, Architekten, Bauherren und Behörden. Richtlinie des BWG, Bundesamt für Wasser und Geologie, Biel 2002

[6] Merkblatt SIA 2018 Überprüfung bestehender Gebäude bezüglich Erdbeben, Schweizerischer Ingenieur- und Architekten-Verein, Zürich 2004.

[7] Wenk, T.: Die neue Norm SIA 269/8 Erhaltung von Tragwerken - Erdbeben, In: D-A-CH-Mitteilungsblatt, Bauingenieur 89 (2014), .Heft 4, S. S2-S3.

[8] Anhang zur Besonderen Bauverordnung I des Kantons Zürich, ZH-Lex 700.21 § 2.9, Änderung vom 6.9.2011.

[9] Beyer, K.; Tondelli, M.; Petry, S.: Rütteltischversuche an einem Gebäude mit Mauerwerks- und Stahlbetonwänden, In: D-A-CH-Mitteilungsblatt, Bauingenieur 88 (2013), Heft 10, S. S 2-S 7.

[10] Bachmann, H.; Koller, M.G.; Somaini, D.: Architektur- und Ingenieurpreis erdbebensicheres Bauen 2012, Stiftung für Baudynamik und Erdbebeningenieurwesen, Dübendorf 2012. www.baudyn.ch

[11] Wenk, T.: Beurteilung der Erdbebensicherheit bestehender Strassenbrücken, Dokumentation, Bundesamt für Strassen, Bern 2005.

[12] Erdbebenversicherung der Basellandschaftlichen Kantonalbank, Versicherteninformation, BLKB, Liestal 2014. https://erdbebenversicherung.blkb.ch/

[13] Wenk, T.; Beyer, K.: Die neue SGEB-Strategie, In: D-A-CH-Mitteilungsblatt, Bauingenieur 88 (2013), Heft 10, S. S 8-S 17 